\title{
Manejo do milheto em pastoreio rotativo para maximizar a taxa de ingestão por vacas leiteiras
}

\author{
[Rotational grazing management in a tropical pasture to maximize the \\ dairy cow's herbage intake rate] \\ J.C. Mezzalira ${ }^{1}$, P.C.F. Carvalho ${ }^{2,5}$, M.F. Amaral ${ }^{3}$, C. Bremm ${ }^{1}$, J.K. Trindade ${ }^{1}$, \\ E.N. Gonçalves ${ }^{1}$, T.C.M. Genro ${ }^{4}$, R.W.S.M. Silva ${ }^{4}$ \\ ${ }^{1}$ Aluno de pós-graduação - Universidade Federal do Rio Grande do Sul - Porto Alegre, RS \\ ${ }^{2}$ Universidade Federal do Rio Grande do Sul - Porto Alegre, RS \\ ${ }^{3}$ Emater-RS - Alegrete, RS \\ ${ }^{4}$ Embrapa CPPSUL - Bagé, RS \\ ${ }^{5}$ Bolsista de produtividade do CNPq
}

\begin{abstract}
RESUMO
Avaliaram-se os efeitos da estratégia de pastoreio rotacionado sobre a dinâmica do rebaixamento do milheto (Pennisetum glaucum (L.) R. Br.) pastejado por vacas leiteiras. Os tratamentos consistiram de um fatorial entre duas alturas pré-pastejo, 60 e $40 \mathrm{~cm}$, e duas pós-pastejo, 20 e $10 \mathrm{~cm}$, alocados em um delineamento em blocos ao acaso com quatro repetições. A estrutura do pasto foi avaliada durante o processo de pastejo a cada 10 minutos, por meio de medições de altura. A taxa de rebaixamento da altura do pasto no tratamento 40-20 apresentou-se relativamente constante ao longo de todo o rebaixamento. O tratamento 60-10 apresentou o maior declínio na taxa de rebaixamento. A taxa de rebaixamento no tratamento 40-10 diminuiu em ritmo mais lento em relação à dos tratamentos com $60 \mathrm{~cm}$ de altura prépastejo. As maiores taxas de ingestão foram observadas nos tratamentos de altura pré-pastejo de $60 \mathrm{~cm}$, e considerou-se como a melhor estratégia de manejo a combinação 60-20cm de altura do pasto.
\end{abstract}

Palavras-chave: estrutura do pasto, Pennisetum glaucum, vacas leiteiras, taxa de ingestão

\begin{abstract}
This study aimed to evaluate the effects of rotational grazing strategies on the dynamics of the grazing pastures of millet (Pennisetum glaucum (L.) R. Br.) grazed by dairy cows. Treatments consisted of two pre-grazing heights (60 and 40cm) and two post-grazing heights $(20$ and $10 \mathrm{~cm})$ allocated in a randomized block design with four replications. The sward height was evaluated during grazing every 10 minutes. The rate of grazing down of the sward height of the pasture on the 40-20 grazing strategy was relatively constant throughout the grazing down. Treatment 60-10 showed the largest decline in the rate of grazing down. The rate of grazing down in the 40-10 treatment decreased with a slower intensity when compared to the treatments with $60 \mathrm{~cm}$ of pre-grazing sward height. The major intake rates were observed in treatments with pre-grazing sward height of $60 \mathrm{~cm}$. The best management strategy was considered the combination of 60-20cm of sward height.
\end{abstract}

Keywords: bite, dairy cows, intake rate, pasture structure, Pennisetum glaucum

\section{INTRODUÇÃO}

O consumo de forragem por ruminantes em pastejo tem sido objeto de inúmeras investigações. Todas reportam numerosos fatores, associados ao animal, ao pasto, ou mesmo fatores abióticos, os quais interferem no processo de busca e captura de forragem pelo animal (Carvalho et al., 2007). Em suma, o consumo de forragem é o produto final do processo de pastejo, cujo manejo pela

Recebido em 30 de maio de 2011

Aceito em 26 de novembro de 2012

E-mail: mezzalirajc@gmail.com 
interferência humana é denominado pastoreio. Isto significa que o pastoreio é uma ferramenta de uso do manejador do pasto com o objetivo de determinar a quantidade e a qualidade da forragem a ser consumida pelo herbívoro. No contexto da produção animal em pasto, as metas de manejo devem proporcionar altos níveis de consumo de forragem (Carvalho et al., 2009).

Tendo em vista que o manejador é quem determina as interações planta-herbívoro no processo de pastejo, entender as decisões comportamentais do animal nas mais discretas escalas é fundamental para subsidiar o manejador nas suas ações. O comportamento dos animais num determinado momento é reflexo das características do pasto moldadas pelo próprio animal via pastejo, e essas decisões, consequentemente, determinarão as características do pasto. E, por outro lado, o animal é influenciado pelas características do pasto criadas por ele mesmo; por exemplo, diante de forte restrição na massa do bocado, o animal tenta compensar via aumento na taxa de bocados, no entanto é penalizado por um "custo fixo" para abrir e fechar a boca (Hirata et al., 2010).

Essas interações em um sistema leiteiro tornamse mais sensíveis, visto que a vaca leiteira tem seu período de alimentação diário reduzido em virtude das ordenhas. Ao se restringir esse período de acesso dos animais ao pasto, corre-se o risco de se impor consequências negativas sobre o consumo diário de forragem. Para tanto, é primordial que a vaca leiteira obtenha alta taxa de ingestão, devido ao curto período disponível para a alimentação, além de ser necessário não expor o animal a horas de pastejo em altas temperaturas, a fim de se evitar o maior custo de mantença exigida pelo animal para regular sua temperatura corporal (Tucker et al., 2008).

O presente trabalho teve por objetivo estudar o manejo do pasto de milheto (Pennisetum glaucum (L.), visando à alta taxa de ingestão de forragem por vacas leiteiras.

\section{MATERIAL E MÉTODOS}

O experimento foi desenvolvido na Embrapa Pecuária Sul, Bagé, RS, $31^{\circ} 20^{\prime} 13^{\prime \prime}$ S e 5406'21" W e altitude de $216 \mathrm{~m}$. Os testes de pastejo foram realizados em março de 2008. Os animais utilizados foram quatro vacas da raça Holandesa, com peso médio de $536,2 \pm 17,8 \mathrm{~kg}$, multíparas e lactantes. A área dos piquetes variou entre $96 \mathrm{e}$ $240 \mathrm{~m}^{2}$ e foi determinada por meio de testes e cálculos prévios. Considerou-se que, em um dia, um bovino adulto pasteja, em média, ao redor de $5-6 \mathrm{~h} / \mathrm{dia}$ e $150 \mathrm{~m}^{2} / \mathrm{animal} / \mathrm{dia}$. Desse modo, calculou-se a área, visando obter o rebaixamento determinado para cada tratamento no decorrer de $60 \mathrm{~min}$ em cada teste de pastejo.

A espécie forrageira utilizada no experimento foi o milheto (Pennisetum glaucum (L.) R. Br.), estabelecido em janeiro de 2008, por plantio direto, com $20 \mathrm{~kg} /$ ha de sementes. A fertilização foi de $100 \mathrm{~kg} /$ ha de adubo formulado $02-20-20$ (NPK), aplicado na semeadura, mais $100 \mathrm{~kg} / \mathrm{ha}$ de nitrogênio em cobertura, na forma de sulfato de amônio.

Os tratamentos consistiram de estruturas de pasto que simularam estratégias de pastoreio rotativo caracterizadas pela combinação de duas alturas de pré, 60 e $40 \mathrm{~cm}$, e duas de pós-pastejo, 20 e $10 \mathrm{~cm}$; dessa combinação formaram-se quatro tratamentos: 60-20; 60-10; 40-20 e 40-10. O delineamento experimental foi o de blocos completos ao acaso, sendo o critério de bloqueamento o turno do dia, manhã e tarde, e com duas repetições no tempo, ciclos de pastejo, totalizando 16 unidades experimentais. $\mathrm{O}$ período de rebaixamento foi de 60 minutos, subdividido em intervalos de 10 minutos, denominados fases 1, 2, 3, 4, 5 e 6. A partir das alturas reais, pré e pós-pastejo, calcularam-se a taxa de rebaixamento da altura em porcentagem da amplitude rebaixada por minuto (\%/min), a proporção da altura a ser rebaixada (\%) e a proporção da altura pré-pastejo, considerando-se a altura pré-pastejo real como $100 \%$.

A determinação da taxa de ingestão foi estimada por meio da técnica da dupla pesagem (Penning e Hooper, 1985), usando-se uma balança de alta precisão $( \pm 0,01 \mathrm{~kg})$. Os animais foram pesados imediatamente antes e após cada teste de pastejo e antes e após o período de perdas metabólicas (Penning e Hooper, 1985), que teve a mesma duração do período de pastejo (60min). Os animais foram equipados com aparelhos de monitoramento automático dos movimentos mandibulares ( Rutter, 2000). Esses aparelhos registram os movimentos mandibulares totais e os distinguem em movimentos de bocados e de não bocados, bem como em tempo de pastejo e 
tempo efetivo de alimentação (descontado os tempos de procura de alimento e sítios preferenciais). Os dados oriundos desses aparelhos foram posteriormente analisados no software GRAZE (Rutter, 2000). Também foi realizado monitoramento visual do comportamento ingestivo. Cada animal foi monitorado por um avaliador previamente treinado, o qual registrava o tempo por estação alimentar, tomando-se o tempo e o número de passos despendidos pelo animal durante o uso de 10 estações alimentares.

Com base nesses dados, determinaram-se: a) número de estações alimentares ocupadas por minuto; b) passos entre estações alimentares visitadas durante o teste de pastejo; c) bocados por estação alimentar, determinados por meio do quociente entre o número total de bocados (obtido pelo aparelho IGER) e a taxa de estações alimentares obtida visualmente; d) taxa de deslocamento - taxa de passos divididos pelo tempo de pastejo efetivo, obtido pelo aparelho IGER; e) tempo por estação alimentar quociente entre a duração total do teste de pastejo, em minutos, e o número total de estações alimentares visitadas, determinado pela taxa de estações alimentares avaliadas visualmente.

A oferta de forragem (g MS/100kg de PV) foi calculada dividindo-se a massa de forragem pelo peso dos animais. A densidade de lotação foi calculada considerando-se o peso corporal médio dos animais entre as condições de pré e de pós-pastejo, somado das perdas metabólicas. Para a determinação da taxa de ingestão, utilizou-se a técnica da dupla pesagem, descrita por Penning e Hooper (1985). Todas as pesagens, pré e pós-pastejo e pré e pós-perdas metabólicas, foram realizadas utilizando-se balança com precisão de $10 \mathrm{~g}$. A taxa de ingestão foi calculada por meio da equação:

$\mathrm{TI}=\frac{(\mathrm{P} 2-\mathrm{P} 1)}{\mathrm{t} 2-\mathrm{t} 1}+\frac{(\mathrm{P} 3-\mathrm{P} 4)}{\mathrm{t} 4-\mathrm{t} 3} \times \frac{(\mathrm{t} 2-\mathrm{t} 1)}{\mathrm{TA}}$,

em que: $\mathrm{TI}=$ taxa de ingestão de forragem; $\mathrm{P} 1 \mathrm{e}$ $\mathrm{P} 2$ = peso dos animais antes e após o pastejo; $\mathrm{t} 1$ e t2 = horário pré e pós-pastejo; P3 e P4 = peso dos animais antes e após as perdas metabólicas; t3 e t4 = horário pré e pós-perdas metabólicas; $\mathrm{TA}=$ tempo efetivo de alimentação.
O total de forragem consumida foi corrigido pelo teor de matéria seca (MS) da forragem, a qual foi estimada com a utilização de um retângulo metálico de $0,127 \mathrm{~m}^{2}$ (31 x $\left.41 \mathrm{~cm}\right)$, com o qual se fizeram dois cortes até a altura de rebaixamento pretendida para cada unidade experimental no pré e pós-pastejo. O resultado do consumo de MS foi utilizado no cálculo da massa do bocado e da taxa de ingestão.

A massa do bocado, $\mathrm{mg}$ de $\mathrm{MS} / \mathrm{kg}^{0,75}$, foi obtida pelo quociente entre a massa de forragem total consumida por $\mathrm{kg}$ de peso metabólico (PM) e o número de movimentos mandibulares de apreensão. A taxa de ingestão, g de $\mathrm{MS} / \mathrm{min} / \mathrm{kg}^{0,75}$, foi obtida pelo quociente entre a massa de forragem total consumida por peso metabólico e o tempo efetivo de alimentação. $\mathrm{O}$ peso metabólico foi determinado pela seguinte equação: $\mathrm{PM}=(\text { peso vivo })^{0,75}$. Foram realizadas 50 medidas de altura do dossel utilizando-se o sward-stick no pré e pós-pastejo em cada teste de pastejo, além de 30 medições da altura do pasto a cada 10 minutos, ao longo do período de rebaixamento.

Para caracterização vertical dos diferentes componentes morfológicos do dossel, fizeram-se dois cortes estratificados a cada $5 \mathrm{~cm}$ da altura do pasto, a partir do nível do solo, com o auxílio de um retângulo metálico de $0,127 \mathrm{~m}^{2}$ (31 x $41 \mathrm{~cm})$. Esses cortes estratificados, somados, compuseram a massa de forragem prépastejo, $\mathrm{kg} / \mathrm{ha}$ de MS. Após cada teste de rebaixamento, foram realizados mais dois cortes de $0,127 \mathrm{~m}^{2}$, em igual procedimento às amostras pré-pastejo, visando à caracterização pós-pastejo. Posteriormente, as amostras foram separadas em lâmina foliar, colmo+bainha e material morto e secas em estufa com ventilação forçada a $55^{\circ} \mathrm{C}$ por 72 horas.

Os dados foram submetidos à análise de variância a 5\% de significância, utilizando-se o procedimento Mixed, segundo o modelo estatístico:

$Y i j k=\mu+\beta i+T j+(\beta T) i j+\gamma k+(T \gamma) j k+\varepsilon i j k$, em que: Yijk representa as variáveis respostas; $\mu$ é uma média inerente a todas as observações; $\beta \mathrm{i}$ é o efeito do i-ésimo bloco; Tj é o efeito do j-ésimo tratamento; $(\beta \mathrm{T}) \mathrm{ij}$ é o efeito aleatório devido à interação do i-ésimo bloco com o j-ésimo tratamento (erro a); $\gamma \mathrm{k}$ é o efeito do 
k-ésimo tempo observado (medida repetida no tempo); (T $\gamma) j k$ é o efeito da interação entre o j-ésimo tratamento e o k-ésimo tempo; e eijk corresponde ao erro aleatório (erro b).

Quando detectadas diferenças entre as médias dos tratamentos, estas foram comparadas pelo teste de Tukey a 5\% de significância. Foram realizadas análises de regressão polinomial até terceira ordem, assim como regressões não lineares, e optou-se por apresentar os resultados pela equação de maior coeficiente de determinação $\left(\mathrm{R}^{2}\right)$, sempre que mais de uma equação de regressão foi significativa $(\mathrm{P}<0,05)$. Quando foram usados testes paramétricos, os pressupostos foram checados e, quando

necessário, foram realizadas transformações. Para a variável taxa de rebaixamento $(\% / \mathrm{min})$, foi realizada transformação com raiz quadrada. Todas as análises foram realizadas com o auxílio do pacote estatístico SAS (Statistical, 2001).

\section{RESULTADOS E DISCUSSÃO}

A altura real média pré-pastejo nos tratamentos de altura inicial pretendida de $60 \mathrm{~cm}$ foi de $60,5 \mathrm{~cm}$, e nos tratamentos de altura pré-pastejo de $40 \mathrm{~cm}$, foi de $42,6 \mathrm{~cm}$. No pós-pastejo, para a altura pós-pastejo pretendida de $20 \mathrm{~cm}$, observouse a altura real de $19,6 \mathrm{~cm}$, e para a altura pós-pastejo pretendida de $10 \mathrm{~cm}$, obtiveram-se $11,7 \mathrm{~cm}$ (Fig. 1).

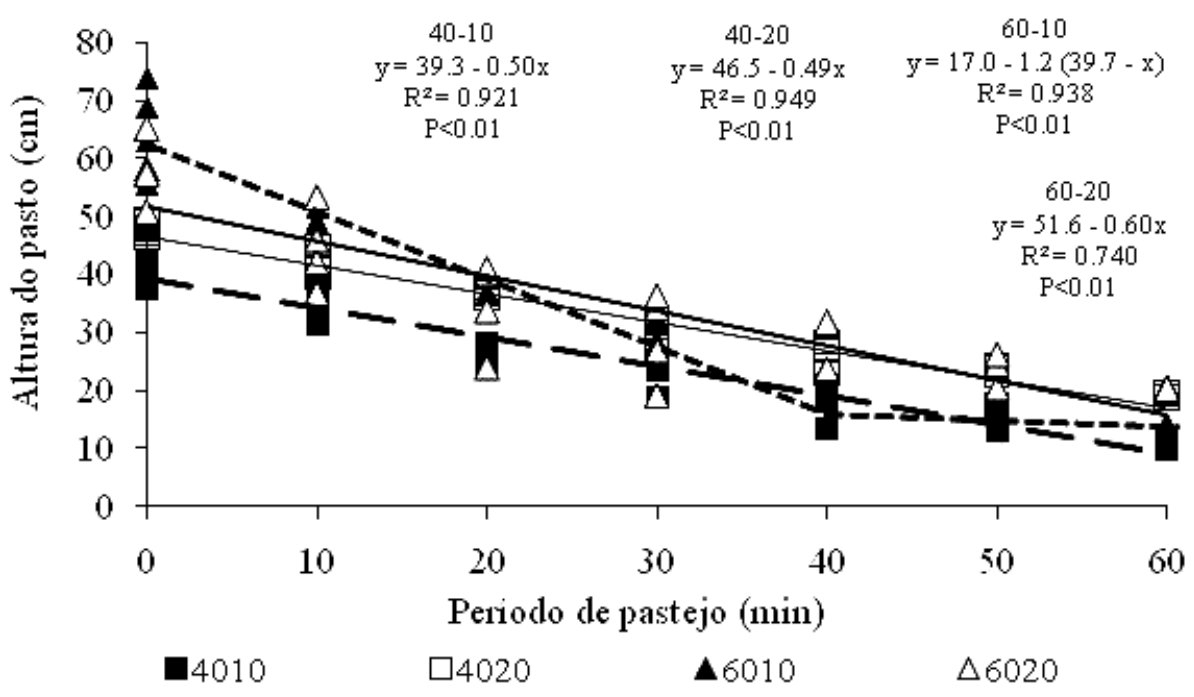

Figura 1. Altura do pasto de milheto pastejado por vacas leiteiras sob manejo rotativo ao longo do rebaixamento (--- 40-10; — 40-20; - - - 60-10; - - 60-20).

Assim como a altura, a massa de forragem pré-pastejo foi superior nos tratamentos de altura pré-pastejo de $60 \mathrm{~cm}$, em comparação aos tratamentos de altura pré-pastejo de $40 \mathrm{~cm}$. Já no pós-pastejo, a massa de forragem no tratamento 60-20 apresentou o maior valor, sendo que os tratamentos 40-20 e 60-10 apresentaram valores intermediários, enquanto no tratamento $40-10$ foi observada a menor massa de forragem no póspastejo (Tab. 1).

A porcentagem de matéria seca média no pré-pastejo foi de $36 \%$, e não foi detectada diferença entre os tratamentos. O percentual de colmos diferiu entre tratamentos $(\mathrm{P}>0,05)$ no pré-pastejo, mas não diferiu no pós-pastejo, assim como o percentual de lâminas foliares verdes no pré-pastejo. O percentual de lâminas foliares verdes no pós-pastejo diferiu entre os tratamentos $(\mathrm{P}<0,05)$. Os tratamentos de altura pós-pastejo de $20 \mathrm{~cm}$ apresentaram o maior percentual de lâminas foliares pós-pastejo, $13 \%$. O tratamento 40-10 apresentou valor intermediário, 10\%, e o tratamento 60-10 apresentou o menor percentual de lâminas no pós-pastejo, 6,5\%. Isso mostra que os animais encontraram uma situação de maior limitação ao consumo no final do período de ocupação neste tratamento. Ou seja, ao ter menor disponibilidade de lâminas foliares, a seletividade por essa parte da planta foi prejudicada. 
Tabela 1. Altura do pasto $(\mathrm{cm})$, massa de forragem $(\mathrm{kg} / \mathrm{ha})$, matéria seca $(\%)$ e proporção de lâminas foliares verdes e colmos $(\%)$ de uma pastagem de milheto sob manejo de pastoreio rotativo

\begin{tabular}{l|ccccc} 
& \multicolumn{5}{c}{ Alturas pré e pós-pastejo (cm) } \\
\cline { 2 - 6 } & $40-10$ & $40-20$ & $60-10$ & $60-20$ & $\mathrm{P}$ \\
\hline Altura pré-pastejo (cm) & $42,0 \mathrm{~b}$ & $43,3 \mathrm{~b}$ & $61,2 \mathrm{a}$ & $59,8 \mathrm{a}$ & $*$ \\
Altura pós-pastejo (cm) & $11,0 \mathrm{~b}$ & $19,0 \mathrm{a}$ & $12,5 \mathrm{~b}$ & $20,2 \mathrm{a}$ & $* *$ \\
Massa de forragem pré-pastejo (kg/ha) & $2432 \mathrm{~b}$ & $2621 \mathrm{~b}$ & $3429 \mathrm{a}$ & $3992 \mathrm{a}$ & $*$ \\
Massa de forragem pós-pastejo (kg/ha) & $1304 \mathrm{c}$ & $1654 \mathrm{~b}$ & $1476 \mathrm{~b}$ & $2080 \mathrm{a}$ & $*$ \\
Matéria seca (\%) & 38,7 & 32,7 & 38,7 & 33,8 & $\mathrm{~ns}$ \\
Colmos pré-pastejo (\%) & $47,6 \mathrm{~b}$ & $47,7 \mathrm{~b}$ & $55,8 \mathrm{a}$ & $59,5 \mathrm{a}$ & $*$ \\
Colmos pós-pastejo (\%) & 67,0 & 74,4 & 81,1 & 71,8 & $\mathrm{~ns}$ \\
Lâminas foliares verdes pré-pastejo (\%) & 40,1 & 33,0 & 38,7 & 33,8 & $\mathrm{~ns}$ \\
Lâminas foliares verdes pós-pastejo (\%) & $10,0 \mathrm{~b}$ & $12,2 \mathrm{ab}$ & $6,5 \mathrm{c}$ & $13,4 \mathrm{a}$ & $* *$ \\
\hline
\end{tabular}

Valores seguidos de letras distintas, na linha, diferem entre si pelo teste Tukey $(\mathrm{P}<0,05)$.

${ }^{\text {ns }}$ não significativo; *significativo a $5 \%$; **significativo a $1 \%$ de probabilidade.

A Fig. 2 mostra a variação na taxa de rebaixamento do pasto, expressa em porcentagem da altura a ser rebaixada/min. $\mathrm{O}$ tratamento 40-20 apresentou a menor taxa de rebaixamento, indicando uma taxa média de rebaixamento de $1,7 \% / \mathrm{min}$, ao longo de todo o período de ocupação. O tratamento $60-10$, com aproximadamente $50 \mathrm{~cm}$ da sua altura inicial removidos, apresentou o maior decréscimo da taxa de rebaixamento. Inicialmente os animais rebaixavam aproximadamente $4 \% / \mathrm{min}$; essa intensidade de rebaixamento diminuiu linearmente até atingir $1 \% / \mathrm{min}$ na última fase do rebaixamento.

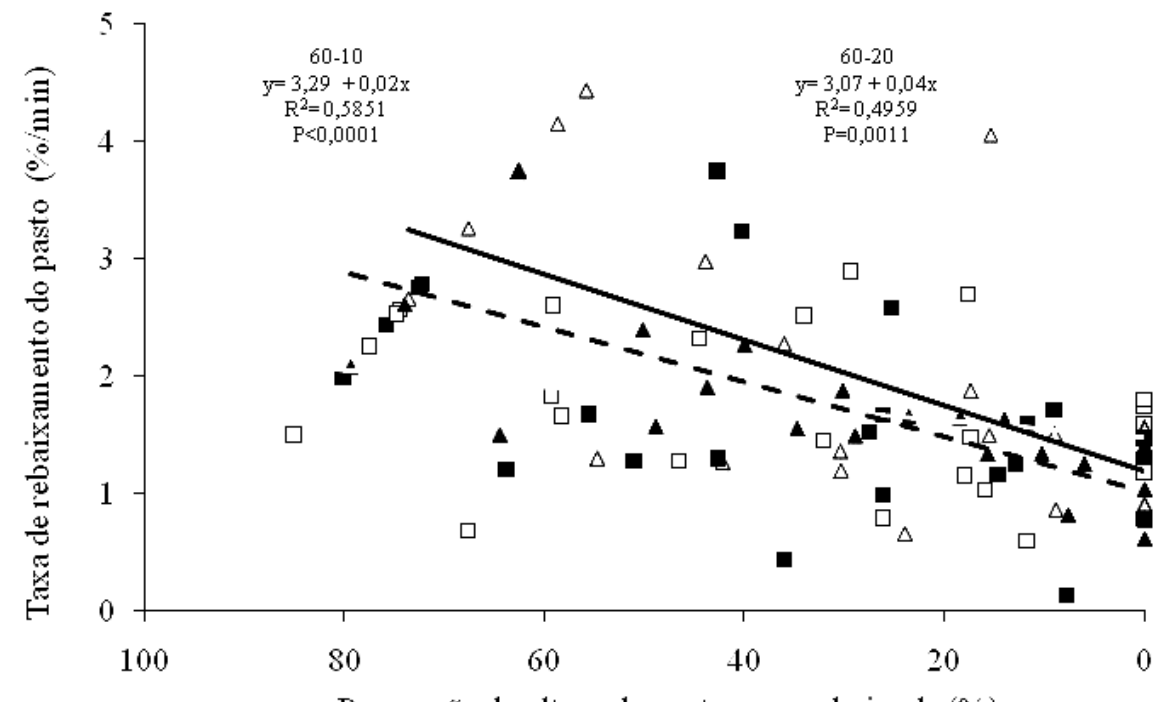

Proporção da altura do pasto a ser rebaixada (\%)

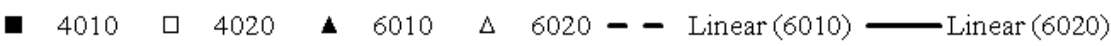

Figura 2. Taxa de rebaixamento de milheto pastejado por vacas leiteiras sob manejo rotativo ao longo do rebaixamento.

Esse estudo da taxa de rebaixamento indica que o rebaixamento do pasto não está diretamente relacionado com a taxa de lotação animal. Ou seja, a disponibilidade de área por animal não apresentou a mesma tendência de resposta observada nos parâmetros que definem a ingestão (massa de bocado e taxa de ingestão), o que demonstra que, ao longo do rebaixamento, os animais podem encontrar estruturas do pasto que conferem impedimentos à colheita de forragem. Tais resultados mostram a importância de se identificar essas estruturas que causam 
impedimentos, a fim de evitar expor os animais a essas situações de limitações ao processo de colheita de forragem, e, com isso, propor metas de manejo com esse intuito e não apenas com base em disponibilidade de área por animal. Como demonstra a Tab. 2, uma mesma disponibilidade de área pode proporcionar respostas totalmente diferentes em razão da altura pré e pós-pastejo.

Sobre os resultados referentes ao tratamento com maior rebaixamento do pasto, 60-10, pode-se observar que, no pós-pastejo, a taxa de rebaixamento do pasto é baixa (Fig. 2), sem alteração na altura do pasto (Fig. 1), com baixo percentual de lâminas foliares verdes disponíveis aos animais (Tab. 1). Essa junção de fatores, somada à redução na taxa de bocados dos animais (Fig. 3), demonstra uma limitação na colheita de forragem quando se impôs maior rebaixamento da altura pré-pastejo. Na tentativa de selecionar folhas, os animais acabam diminuindo a massa do bocado e a taxa de bocados, o que leva a uma drástica redução na taxa de ingestão com altas intensidades de rebaixamento (Amaral et al., 2013; Fonseca et al., 2012).

A massa de bocado mostrou um padrão de resposta em que os tratamentos com maior altura pós-pastejo, $20 \mathrm{~cm}$, permitiram aos animais colher maiores massas de bocado, $12,9 \mathrm{mg} / \mathrm{boc} / \mathrm{kg}^{0,75}$, em comparação com a massa de bocado da altura pós-pastejo de $10 \mathrm{~cm}$, $11,4 \mathrm{mg} / \mathrm{boc} / \mathrm{kg}^{0,75}$. A maior taxa de ingestão foi observada no tratamento 60-20, sendo registrados $0,53 \mathrm{~g}$ de $\mathrm{MS} / \mathrm{kg}^{0,75} / \mathrm{min}$. Os demais tratamentos não diferiram entre si, com média de $0,45 \mathrm{~g}$ de $\mathrm{MS} / \mathrm{kg}^{0,75} / \mathrm{min}$ (Tab. 2).

Tabela 2. Massa do bocado (MB), taxa de bocados (TB), tempo por bocado (s), taxa de ingestão (TI), movimentos mandibulares totais, oferta de forragem, disponibilidade de área durante o rebaixamento dos pastos de milheto submetidos a diferentes combinações de alturas pré e pós-pastejo

\begin{tabular}{l|ccccc} 
& \multicolumn{5}{c}{ Alturas pré e pós-pastejo $(\mathrm{cm})$} \\
\cline { 2 - 6 } & $40-10$ & $40-20$ & $60-10$ & $60-20$ & $\mathrm{P}$ \\
\hline Massa do bocado $\left(\mathrm{mg} / \mathrm{boc} / \mathrm{kg}^{0,75}\right)$ & $11,9 \mathrm{~b}$ & $12,6 \mathrm{a}$ & $11,0 \mathrm{~b}$ & $13,1 \mathrm{a}$ & $*$ \\
Taxa de bocados $(\mathrm{boc} / \mathrm{min})$ & $43,3 \mathrm{a}$ & $36,3 \mathrm{~b}$ & $39,6 \mathrm{a}$ & $37,8 \mathrm{~b}$ & $*$ \\
Taxa de ingestão $\left(\mathrm{g} \mathrm{MS} / \mathrm{kg}^{0,75} / \mathrm{min}\right)$ & $0,41 \mathrm{~b}$ & $0,45 \mathrm{~b}$ & $0,48 \mathrm{ab}$ & $0,53 \mathrm{a}$ & $* *$ \\
Movimentos mandibulares $\left(\mathrm{n}^{\circ} / \mathrm{min}\right)$ & $73,0 \mathrm{a}$ & $71,5 \mathrm{a}$ & $72,7 \mathrm{a}$ & $73,5 \mathrm{a}$ & $\mathrm{ns}$ \\
Oferta de forragem $\left(\mathrm{m}^{3} / 100 / \mathrm{kg}\right)$ & $2,9 \mathrm{~b}$ & $4,5 \mathrm{a}$ & $3,2 \mathrm{ab}$ & $3,2 \mathrm{ab}$ & $*$ \\
Disponibilidade de área $\left(\mathrm{m}^{2} / \mathrm{animal}\right)$ & $37,5 \mathrm{~b}$ & $50,7 \mathrm{a}$ & $27,5 \mathrm{c}$ & $29,0 \mathrm{bc}$ & $* *$ \\
Disponibilidade de área $\left(\mathrm{m}^{2} / 100 \mathrm{~kg} \mathrm{PV}\right)$ & $6,87 \mathrm{ab}$ & $9,15 \mathrm{a}$ & $5,25 \mathrm{~b}$ & $5,01 \mathrm{~b}$ & $* *$ \\
\hline
\end{tabular}

Valores seguidos de letras distintas, na linha, diferem entre si pelo teste de Tukey $(\mathrm{P}<0,05)$.

${ }^{\text {ns }}$ não significativo; *significativo a $5 \%$; **significativo a $1 \%$ de probabilidade.

Quando são comparados os tratamentos que proporcionaram maiores taxas de ingestão durante o rebaixamento, $60-10$ e $60-20 \mathrm{~cm}$, podese observar que o tratamento 60-10 impôs algumas limitações aos animais, visto que eles aparentemente "desistiram" de pastejar ao final do período de ocupação (Ribeiro Filho et al., 2003; Amaral et al., 2013), pela redução da taxa de bocados (Fig. 3), provavelmente em razão do baixo percentual de lâminas foliares disponíveis (Tab. 1). Além disso, em termos produtivos, a pastagem rebaixada até menores alturas necessita de maior período de descanso, ou seja, maior intervalo de tempo entre os pastoreios em um sistema rotacionado, o que resultará em prejuízos para a produção animal.
O tratamento 60-10 apresentou a maior variação da taxa de bocados ao longo do rebaixamento. No início, os animais apresentaram taxa de $25 \mathrm{boc} / \mathrm{min}$, taxa que aumenta até $43 \mathrm{boc} / \mathrm{min}$, momento em que já haviam sido rebaixados aproximadamente $75 \%$ da altura pretendida. Em seguida, diminuiu-se até atingir $36 \mathrm{boc} / \mathrm{min}$ no final do rebaixamento (Fig. 3). Segundo Stakelum e Dillon (2007), a taxa de bocados diminui à medida que a qualidade da estrutura do pasto é deteriorada, fato que pode ser observado no final do período de ocupação. No método de pastoreio rotacionado, essa mudança de "qualidade" da estrutura ocorre principalmente quando o animal passa a pastejar uma nova camada do pasto (Baumont et al., 2004), geralmente caracterizada por maior percentual de colmos. 


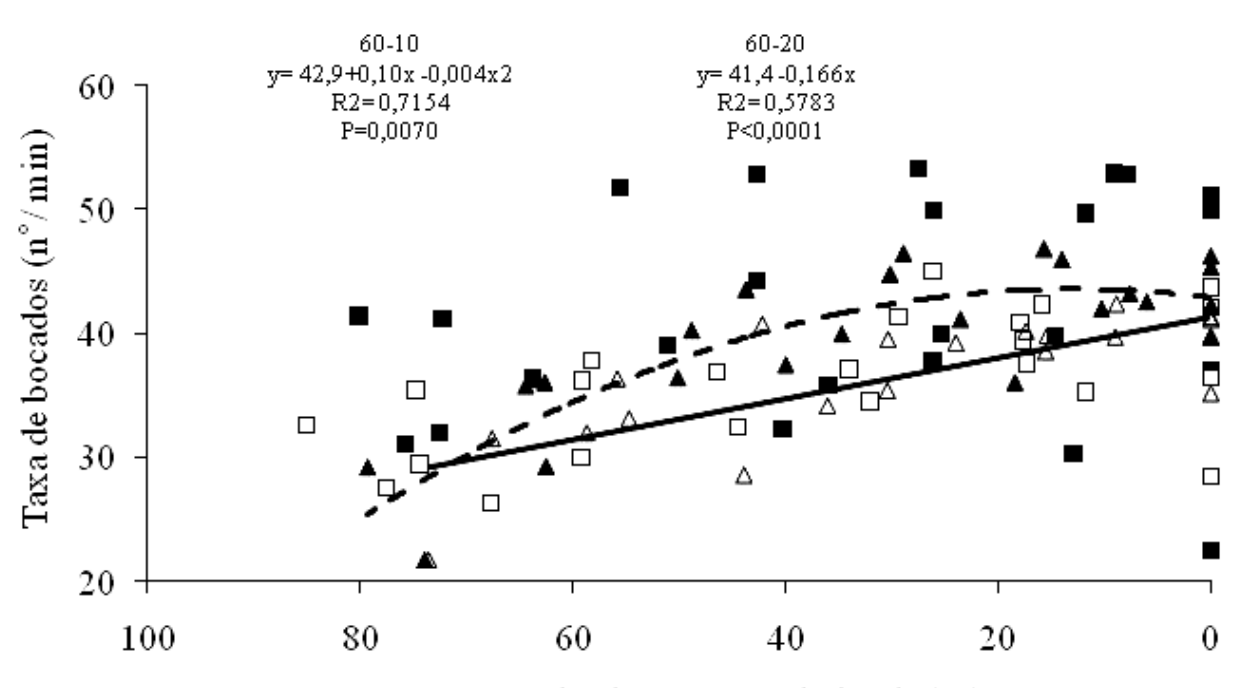

Proporção da altura a ser rebaixada $(\%)$

- $4010 \square \quad 4020 \Delta 6010 \Delta 6020-$ Polinômio (6010) $\longrightarrow$ Linear (6020)

Figura 3. Taxa de bocados ( $\left.{ }^{\circ} / \mathrm{min}\right)$ em função da proporção da altura a ser rebaixada por vacas leiteiras sob manejo rotativo ao longo do rebaixamento de pastos de milheto.

A taxa de bocados ao longo do período de rebaixamento foi semelhante nos tratamentos de altura pós-pastejo de $20 \mathrm{~cm}$. No tratamento $40-20$, houve aumento na taxa de bocados de 29boc/min, no início do rebaixamento até 40boc/min ao final desse período, semelhante ao que ocorre no tratamento 60-20, em que animais aumentam a taxa de bocados de 25 até $41 \mathrm{boc} / \mathrm{min}$ entre o início e o fim do período de rebaixamento. Observou-se o aumento da taxa de bocados (Fig. 3) no tratamento 60-20 à medida que o dossel do pasto foi rebaixado, sendo que os animais aumentaram a taxa de bocados de 25 até $41 \mathrm{boc} / \mathrm{min}$ entre o início e o fim do período de rebaixamento (Tab. 2). Esse comportamento demonstra que as menores alturas do pasto resultaram em diminuição da massa do bocado, e, dessa forma, os animais alocaram maior proporção de movimentos mandibulares para apreensão de forragem, visto que animais têm taxa de movimentos mandibulares constantes (Ungar, 1996). Da mesma fora, ao se deparar com estruturas de pasto drasticamente rebaixadas, os animais tomam bocados de menores massas. Isto lhes permite dedicar maior número de movimentos mandibulares para tomar bocados (Ungar, 1996). Por isso foi observado maior taxa de bocados nos tratamentos de altura pós-pastejo de $10 \mathrm{~cm}$ (Tab. 2). Inversamente aos resultados de taxa de bocados, as maiores massas de bocado foram registradas nos tratamentos que mantiveram a alturas pós-pastejo de $20 \mathrm{~cm}$. Nessas situações, os animais consomem bocados com massas de aproximadamente $13 \mathrm{mg}$ de $\mathrm{MS} / \mathrm{kg}^{0,75}$, superiores aos valores de $11,45 \mathrm{mg}$ de $\mathrm{MS} / \mathrm{kg}^{0,75}$ observados nos tratamentos de altura de saída de $10 \mathrm{~cm}$.

No processo de pastejo, a colheita de forragem é o equivalente ao máximo de interação plantaanimal. Quando se força o animal a pastejar em camadas inferiores do dossel do pasto, a massa do bocado é reduzida, devido ao fato de que o animal tenta expressar seletividade por folhas (Benvenutti et al., 2006; Drescher et al., 2006) cada vez mais escassas em meio a componentes menos desejados (colmos, material morto) da planta (Penning et al., 1991). Semelhante resultado já havia sido observado por Barrett et al. (2001), que verificaram declínio na massa de bocados colhida por vacas ao longo do dia, em pastos manejados pelo método de pastoreio rotativo. Essa combinação de altura pré e póspastejo de $60-20 \mathrm{~cm}$ se comportou como a meta de manejo que aliou alta massa de forragem no pré-pastejo, permitindo alta massa de bocado e alta taxa de ingestão. Ao se observar diminuição gradual da taxa de rebaixamento e aumento 
gradual na taxa de bocados, pode-se inferir que são alterações sutis e normais em razão do rebaixamento do pasto, e não alterações em que os animais desistem de pastejar devido à drástica alteração da estrutura do pasto (Ribeiro Filho et al., 2003; Amaral et al., 2013). Dessa forma, sugere-se que a pastagem de milheto seja manejada com altura pré e pós-pastejo de 60 e $20 \mathrm{~cm}$, respectivamente.

\section{CONCLUSÕES}

Para metas de manejo do pastoreio que visem aliar colheita de alta massa de bocado e proporcionar alta taxa de ingestão, recomendamse $60 \mathrm{~cm}$ de altura no pré-pastejo e $20 \mathrm{~cm}$ no póspastejo.

\section{REFERÊNCIAS}

AMARAL, M.F.; MEZZALIRA, J.C.; BREMM, C. Sward structure management for a maximum shortterm intake rate in annual ryegrass. Grass Forage Sci., v.68, p.271-277. 2013.

BARRETT, P.D.; LAIDLAW, A.S.; MAYNE, C.S. et al. Pattern of herbage intake rate and bite dimensions of rotationally grazed dairy cows as sward height declines. Grass Forage Sci., v.56, p.362-373, 2001.

BAUMONT, R.; COHEN-SALMON, D.; PRACHE, S. et al. A mechanistic model of intake and grazing behaviour in sheep integrating sward architecture and animal decisions. Anim. Feed. Sci. Tech., v.112, p.528, 2004.

BENVENUTTI, M.A.; GORDON, I.J.; POPPI, D.P. The effect of the density and physical properties of grass stems on the foraging behaviour and instantaneous intake rate by cattle grazing an artificial reproductive tropical sward. Grass Forage Sci., v.61, p.272-281, 2006

CARVALHO, P.C.F.; KOZLOSKI, G.V.; RIBEIRO FILHO, H.M.N. et al. Avanços metodológicos na determinação do consumo de ruminantes em pastejo. Rev. Bras. Zootec., v.36, supl. especial, p.151-170, 2007.

CARVALHO, P.C.F.; TRINDADE, J.K.; MEZZALIRA, J.C. et al. Do bocado ao pastoreio de precisão: compreendendo a interface planta-animal para explorar a multi-funcionalidade das pastagens. Rev. Bras. Zootec., v.38, p.109-122, 2009.
DRESCHER, M.; HEITKONIG, I.M.A.; RAATS, J.G.; PRINS, H.H.T. The role of grass stems as structural foraging deterrents and their effects on the foraging behaviour of cattle. Appl. Anim. Behav. Sci., v.101, p.10-26, 2006

FONSECA, L.; MEZZALIRA, J.C.; BREMM, C. Management targets for maximising the short-term herbage intake rate of cattle grazing in Sorghum bicolor. Livest. Sci., v.145, p.205-211, 2012.

HIRATA, M.; KUNIEDA, E.; TOBISA, M. Short-term ingestive behaviour of cattle grazing tropical stoloniferous grasses with contrasting growth forms. J. Agr. Sci., v.148, p.615-624, 2010.

PENNING, P.D.; HOOPER, G.E.N. A evaluation of the use of short-term weight changes in grazing sheep for estimating herbage intake. Grass Forage Sci., v.40, p.79-84, 1985.

PENNING, P.D.; PARSONS, A.J.; ORR, R.J. et al. Intake and behaviour responses by sheep to changes in sward characteristics under continuous stocking. Grass Forage Sci., v.46, p.15-28, 1991.

RIBEIRO FILHO H.M.N.; DELAGARDE, R.; PEYRAUD, J.L. Inclusion of white clover in stripgrazed perennial ryegrass swards: herbage intake and milk yield of dairy cows at different ages of sward regrowth. Anim. Sci., v.77, p.499-510, 2003.

RUTTER, S.M. Graze: A program to analyze recordings of the jaw movements of ruminants. Behav. Res. Meth., Instrum. Comp., v.32, p.86-92, 2000.

STATISTICAL analysis system - SAS. SAS/STAT user's guide: statistics. 4.ed. Version 8.2. Cary: 2001, v.2. $943 \mathrm{p}$.

STAKELUM, G.; DILLON, P. The effect of grazing pressure on rotationally grazed pastures in spring/early summer on the performance of dairy cows in the summer/autumn period. Irish. J. Agr. Food. Res., v.46, p.29-46, 2007.

TUCKER, C.B.; ROGERS, A.R.; SCHÜTZ, K.E. Effect of solar radiation on dairy cattle behaviour, use of shade and body temperature in a pasture-based system. Appl. Anim. Behav. Sci., v.109, p.141-154, 2008.

UNGAR, E.D. Ingestive behaviour. In: HODGSON, J.; ILLIUS, A. (Eds.). The ecology and management of grazing systems. Wallingford: CABI, 1996. p.185-218. 